\title{
Research on Remote Condition Monitoring System in Coal Mine
}

\author{
http://dx.doi.org/10.3991/ijoe.v10i1.3197 \\ Wang Wenxing \\ North China University of Water Resources and Electric Power, Zheng zhou P.R. China
}

\begin{abstract}
In this paper, the signal sources of communication system are described in coal mine firstly, and then a kind of remote condition monitoring system based on the OFDM technology is presented. This system makes wire communication combined mutually with wireless communication by tandem adapter. Wireless communication is used in mining area or in the time of sudden accident, Power line or fiber-optic is used in main lane. This system makes use of the advantage of OFDM technology to provide many kinds of access service in mine. There are different grades and different rates for many consumers at the same time in these access services. The different modulation methods of subcarries in this system increase flexibility and adaptability. The system not only fulfils the usual demand of produce communication monitoring, but also considers sudden accident, attains the state where the mining area arrives where the signal transports. This paper analyzed feasibility of the system based on some simulation tests finally.
\end{abstract}

Index Terms-sensor networks, remote sensing, OFDM, coal mine

\section{INTRODUCTION}

The coal produce proceeds under the well generally, and the circumstances in mine are complicated. The drifts and roadways are narrow and small, personals move frequently, work complicated. Along with modern technical development in coal, urgent demand contains a quick, accurate, flexibility mine communication system. In conventional equipment monitoring, artificially is used by accumulated experience, the accuracy is not high enough.[1]. In order to solve difficult such as maintenance work, management issues inconvenience, it is an effective way to establishing a remote monitoring system [2]. Orthogonal Frequency Division Multiplexing (OFDM) is a multi-carrier technique that has recently received considerable attention for high speed communication. OFDM has emerged as one of the primary candidates for the Fourth Generation (4G) wireless communication systems [3]. This paper analyses the signal sources of communication and remote condition monitoring system in mine firstly, and then presents a kind of multiple access system based on the OFDM technology in mine.

\section{COMMUNiCATION SYSTEM}

The signal sources of monitoring and communication underground system in coal mine not only come from single parameter monitoring system, but also come from many parameters in one aspect and many parameters in various aspects. The transmission signals of the system in mine include switching-signal, analog signal, digital sig- nal and so on. Distribution range of transmission rate is wide from monitoring transmission date of $100 \mathrm{bps}$ sensor to picture transmission of $216 \mathrm{Mbps}$ each path; Modulation methods in mine include baseband modulation, Amplitude Modulation, Frequency Modulation, Frequency Shift Keying, Phase Shift Keying and so on. Manual measurement exist many problems, not real-time monitoring of data changes. Modern machineries have complex structure involving many areas of technology; the maintenance is difficult with heavier tasks [4]. Remote automatic monitoring system can effectively solve these problems [5].

Fig. 1 shows the remote condition monitoring system. Remote monitoring system uses three level tandem structures: the control center, monitoring unit and monitoring sensor. This system may transmit the single of measuring and monitoring such as the voltage, the velocity, and water press, the monitors for the production. Wireless and wire signal receiving equipments send signal to plant control centre through monitoring unit.

This system employs wireless communication for movement consumer and power line communication (PLC) for stationary dot consumer, makes wire communication combined mutually with wireless communication by tandem adapter.

However, the investment of optical fiber communication is relatively large, it is difficult installation in shortdistance communication, the mountains and geographically dispersed, etc., and protection and maintenance are also difficulties.

The sub-carrier transmission mode is introduced to Multi-carriers. Data resources are occupied during the user send or receive which means that multiple users can efficiently share the same channel, improved resource utilization.

This system employs wireless communication for movement consumer and power line communication (PLC) for stationary dot consumer, makes wire communication combined mutually with wireless communication by tandem adapter.

OFDM can be used as modulation / demodulation for PLC and wireless communication. This system may transmit the single of measuring and monitoring such as the air density, the wind velocity, and dust density, the monitors for the production equipments, etc.. Unlike power line applications, signal transmitted over wireless channels may suffer power losses, shadowing effects, multipath fading, and interference from many sources. As a result, signals at the receiver are subject to high error rates and the required signal to noise rate (SNR) to meet the quality of service (QoS) is usually higher than power line systems. Therefore, robust transmission techniques are 


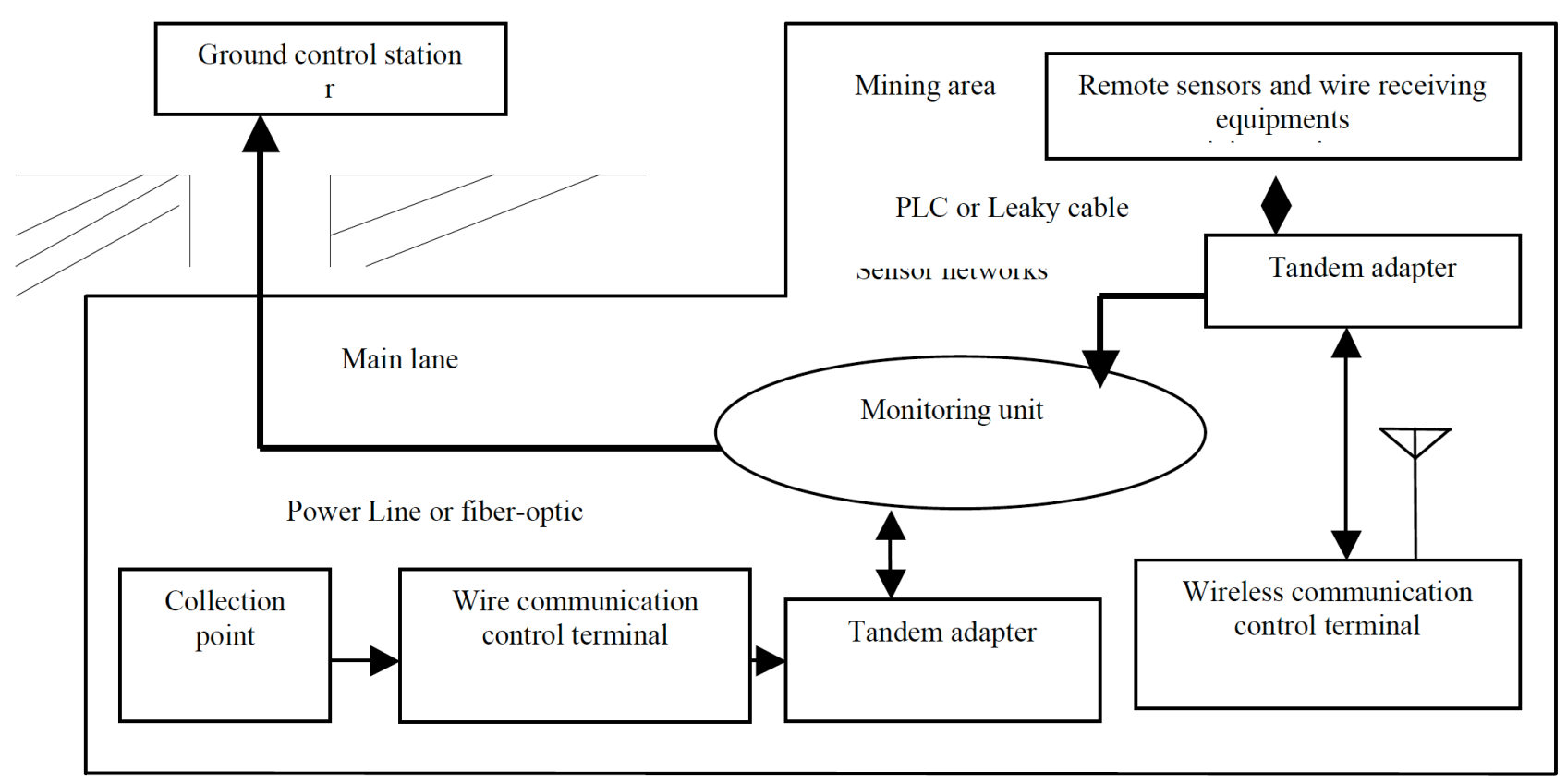

Figure 1. Functional block diagram of the system

required to reduce the detrimental effects caused by wireless channels.

\section{SYSTEM ANALYSES}

Remote monitoring system usually consists of front-end acquisition, signal transmission, remote monitoring centers. [6]。 The information of this system from remote sensors are collected by the monitoring unit, remote operation of all switches is achieve through the monitoring unit. Monitoring module is installed in the side of the standard control panel, including the power supply module, management, communication module, remote module, and remote measurement module.

The tandem adapter includes core single chip, data acquisition, data processing, keyboard input, comparative judgments, display, and alarm equipment. Block diagram of tandem adapter is shown as Fig.2. All orders of the remote monitoring system is kept apart in remediate relay through the screen, and then output to the operation of circuit switching.

The communication means of tandem adapter connections among has microwave, optical fiber, power line, and others. Flow diagram of control is shown as Fig.3. After system architecture is completed, because the modem is plug and play device, as long as the network connection is correct, then data can be normal transmitted[7]。

The subsurface communication in mine develops slowly because of the special environment condition.

Power Line Communication (PLC) is a kind of technique that exploits the available low pressure power line wire net to act as the medium to transmit the signal of data, language and image [8]. The power line has the following characteristics: it can wire in all directions, distribute extensively, transfer signal with the work need and needn't arrange line again. There are a few problems in using this system to transmit the signal as communication channel: (1) Character of time-varying; (2) Complexity of the interference; (3) High noise; (4) Multipath interference.
The sub-carriers are allowed to overlap but they are still mathematically orthogonal to each other.

This makes the OFDM spectrally efficient compared to a conventional multi-carrier system. The sum of subcarriers consist OFDM symbols and sub-carriers are modulated by PSK or QAM. The sample of an OFDM symbol can be written as [9]:

$$
\begin{aligned}
& s(t)=\operatorname{Re}\left\{\sum_{i=0}^{N-1} d_{i} \operatorname{rect}\left(t-t_{s}-T / 2\right)\right. \\
& \left.\cdot \exp \left[j 2 \pi\left(f_{c}+i / T\right)\left(t-t_{s}\right)\right]\right\} \\
& \left(t_{s} \leq t \leq t_{s}+T\right) \\
& \mathrm{s}(\mathrm{t})=0 \quad \mathrm{t}<\mathrm{ts} \text { and } \mathrm{t}>\mathrm{T}+\mathrm{ts}
\end{aligned}
$$

where $\mathrm{N}$ is the number of sub-carrier, fc is the first sub carrier frequency, di denotes transmitted data symbol on the carrier.

The symbol length of $s(t)$ is T. Being used as a kind of modulation method, the technique of OFDM can also combine together with various multiple address very easily.

It provides the connection for the multi-user into the serve at the same time. The main multiple access includes FDMA, CDMA and TDMA. Many consumers in the different site may be connected with

However, the investment of optical fiber communication is relatively large, it is difficult installation in shortdistance communication, the mountains and geographically dispersed, etc., and protection and maintenance are also difficulties.

Control center and monitoring should be able to display electrical equipment condition and operating parameters 


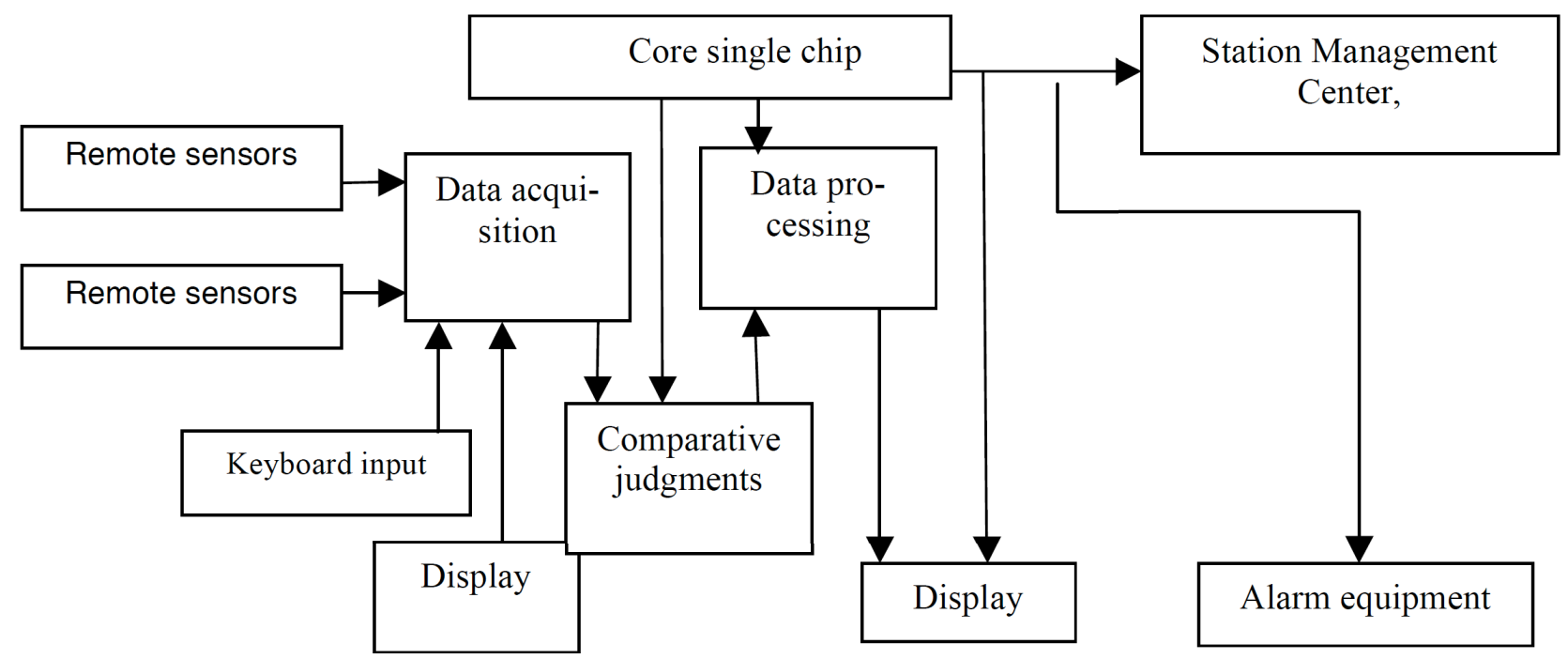

Figure 2. Diagram of Tandem Adapter

properly within its control monitored all the time for power plant. For monitoring equipment with intelligent interfaces, the equipment should be used to provide interface functions to achieve its software. System should have the sequential logic control to monitoring equipment.

The sub-carrier transmission mode is introduced to Multi-carrieris. Data resources are occupied during the user send or receive which means that multiple users can efficiently share the same channel, improved resource utilization. each other on multiple addresses at the same time. In multi-code CDMA system, one of spread spectrum code is selected to send directly transmission data when the consumer needs in mining area. In modern communication, SS (Spread Spectrum) is extensive and became an important means, because of its high noise resistance, low interception probability and good security [10].

Many spread spectrum pseudo noise code can be used at the same time. CDMA has the capability multiple addresses. The main reason is that sequence of spread spectrum has good characteristics of auto-correlation, crosscorrelation and partial correlation [6]

One of the other benefits of spreading in the timedomain in each individual sub-channel is the high flexibility to handle service-on-demand (SOD) for multimedia applications in mine. Different combination of subchannels and code-word channels can be assigned to difference subscribers with different transmission rate requirements.

The main ways of wireless communication system primarily include through-the-earth communication system, inductive communication system and leaky communication system, etc.. Microwave belong to electromagnetic wave, mode of propagation is rectilinear. propagation. Microwave still contain its special action in the nature accident and artificial fault, the action of the microwave communication mustn't be neglected. The through-theearth communication is a kind of communication method that regards earth ground as the electromagnetic wave spreads medium, and radio wave penetrate the earth ground. The through-the-earth communication system is applicable to the short distance and relief assistance communication system. Inductive communication transmits the signal through definite line of induction, cost of the system is low, the equipment is simple, but the distance of signal transmission is nearer. The leaky communication can make use of the relay station to realize the longdistance transmission

\section{TEST AND CONCLUSION}

We tested the data communication based on MULTIPLE ACCESS with the OFDM modulation method. The test of throughput is as follows (unit:Mbps) :

(1) The signal was coupled with one of the phases current:

A- phase: Average throughput: 1.028; Biggest throughput: 1.311; Minimum throughput: 0.296

B- phase: Average throughput: 1.122; Biggest throughput: 1.396; Minimum throughput: 0.275

The result of the test displayed that system based on MULTIPLE ACCESS with the OFDM modulation method feasibility. We can observe that the number of average throughput is distributed mainly at $1 \mathrm{Mbps} \sim 2 \mathrm{Mbps}$ and the signal coupled with one of the phases current can be sent to the destination.

This paper analyzes the signal sources of communication system in mine firstly, and then put forward the remote condition monitoring system in mine based on OFDM. The system not only satisfies the usual demand of remote produce communication monitoring, but also considers dealing sudden accident. This system makes wire communication combine with wireless communication by tandem adapter. Wireless communication is used in mining area and sudden accident. Since variety of the modulation methods, we can choose appropriate modulation method according to equipment transmission signals in order to resolve the compatibility problem of whole system. The experiment was introduced finally to realize the high-speed data transmission, and the result shows that it is viable. 


\section{RESEARCH ON REMOte CONDITION MONITORING System In COAL Mine}

\section{REFERENCES}

[1] CAO Yong. Remote Fault Diagnosis System of Engineering Machinery. Microprocessors, 2012,33(3):58-60

[2] HE Qing - hua. Design and Implementation of Remote Engineering Equipment Monitoring System. JOURNAL OF ZHENGZHOU UNIVERSITY(ENGINEERING SCIENCE), 2009,30(2):66-70

[3] Peng Ling. Third Generation Mobile Communication Technology. Publishing House of Electronics Industry.2003.2. p:396

[4] ZHANG Qi. Implementation of engineering machine remote diagnosis and maintenance system based on internet, JOURNAL OF PLA UNIVERSITY OF SCIENCE AND TECHNOLOGY (NATURAL SCI ENCE EDITION), 2009,10(z1):49-51

[5] TANG Jiangyang.Research on Remote Automatic Monitoring System in the Engineering Construction. China's Manganese Industry,2012,30(1):38-40

[6] QU Guang-jian. Remote video surveillance technology research and application in engineering blasting. Engineering Blasting, 2012, 18(3):81-84
[7] LIU Linan, Applications of remote video monitoring system in the construction of over - fiver tunnel. SHANGHAI CONSTRUCTION SCIENCE \& TECHNOLOGY 2010(3):43-45

[8] Dr John Newbury. Communication Requirements and Standards for Low Voltage Mains Signaling, IEEE Transaction Power Delivery, 1998,13 (1) pp: 46-53 http://dx.doi.org/10.1109/61. $\underline{660847}$

[9] Wang Bingjun. Spread Spectrum Communication: Tianjin University Publishing 1993. p:5

[10] Cai Jueping, Li Jianxin Study CDMA Random Multiple Access Technique: Communications Technology. No.112, Totally. pp:2022

\section{AUTHOR}

Wang Wenxing is with North China University of Water Resources and Electric Power, Zheng zhou, P.R. China, 450011.

Submitted 16 September 2013. Published as re-submitted by the author 23 January 2014. 